\title{
Effects of Oxytocin on Neural Response to Facial Expressions in Patients with Schizophrenia
}

\author{
Na Young Shin', Hye Yoon Park', Wi Hoon Jung', Jin Woo Park ${ }^{2}$, Je-Yeon Yun², Joon Hwan Jang 1,2, \\ Sung Nyun Kim², Hyun Jung Han ${ }^{3}$, So-Yeon Kim³, Do-Hyung Kang ${ }^{1,2}$ and Jun Soo Kwon*, ${ }^{*, 2,3}$ \\ 'Institute of Human Behavioral Medicine, SNU-MRC, Seoul, Republic of Korea; ${ }^{2}$ Department of Psychiatry, Seoul National University College of \\ Medicine, Seoul, Republic of Korea; ${ }^{3}$ Department of Brain and Cognitive Sciences, College of Natural Sciences, Seoul National University, \\ Seoul, Republic of Korea
}

\begin{abstract}
Impaired facial emotion recognition is a core deficit in schizophrenia. Oxytocin has been shown to improve social perception in patients with schizophrenia; however, the effect of oxytocin on the neural activity underlying facial emotion recognition has not been investigated. This study was aimed to assess the effect of a single dose of intranasal oxytocin on brain activity in patients with schizophrenia using an implicit facial emotion-recognition paradigm. Sixteen male patients with schizophrenia and 16 age-matched healthy male control subjects participated in a randomized, double-blind, placebo-controlled crossover trial at Seoul National University Hospital. Delivery of a single dose of $40 \mathrm{U}$ intranasal oxytocin and the placebo was separated by I week. Drug conditions were compared by performing a region of interest $(\mathrm{ROI})$ analysis of the bilateral amygdala on responses to the emotion recognition test. It was found that nasal spray decreased amygdala activity for fearful emotion and increased activity for happy faces. Further, oxytocin elicited differential effects between the patient and control groups. Intranasal oxytocin attenuated amygdala activity for emotional faces in patients with schizophrenia, whereas intranasal oxytocin significantly increased amygdala activity in healthy controls. Oxytocin-induced BOLD signal changes in amygdala in response to happy faces was related to attachment style in the control group. Our result provides new evidence of a modulatory effect of oxytocin on neural response to emotional faces for patients with schizophrenia. Future studies are needed to investigate the effectiveness of long-term treatment with intranasal oxytocin on neural activity in patients with schizophrenia.
\end{abstract}

Neuropsychopharmacology (2015) 40, 1919-1927; doi:10.1038/npp.2015.4I; published online 4 March 2015

\section{INTRODUCTION}

Schizophrenia is characterized by impairments in cognitive function, emotional processing, and social functioning. Patients with schizophrenia suffer from positive symptoms, such as hallucinations and delusions, and negative symptoms, such as reduced emotional expression and anhedonia.

Converging evidence indicates that the identification of facial emotion is impaired in schizophrenia (Morris et al, 2009). Studies suggested the involvement of the amygdala, which has a critical role in emotional processing (Fitzgerald et al, 2006), in deficient emotional recognition in schizophrenia (Aleman and Kahn, 2005; Gur et al, 2002; Michalopoulou et al, 2008). In neuroimaging studies, amygdala activity evoked by a fearful face was attenuated relative to that evoked by a neutral face in patients with schizophrenia (Taylor et al, 2012). However, recent neuroimaging studies suggest that this is due to an increased activation in response to the neutral faces rather than to a decreased response to

\footnotetext{
*Correspondence: Professor JS Kwon, Department of Psychiatry and Department of Brain and Cognitive Sciences, Seoul National University, 28 Yeongon-dong, Chongno-gu, Seoul II0-744, Republic of Korea, Tel: +82 22072 2972, Fax: +82 2747 9063, E-mail: kwonjs@snu.ac.kr Received 10 October 2014; revised 19 January 2015; accepted 20 January 2015; accepted article preview online 10 February 2015
}

fearful faces (Hall et al, 2008; Holt et al, 2006). Such findings indicate that increased social anxiety or social fear in patients with schizophrenia results in the tendency to perceive neutral faces as aversive or salient (Morris et al, 2009; Taylor et al, 2012).

Recent studies have shown that intranasal administration of oxytocin modulated various social behaviors including emotion recognition, empathy, social memory, trusting behavior, generosity, and cooperation in humans (See the review article by Bartz et al, 2011). Oxytocin has been shown to enhance the ability to identify fearful and happy facial expressions, although the results are inconsistent across studies (See the review article by Shahrestani et al, 2013). Furthermore, neuroimaging studies have shown that intranasal oxytocin attenuated neural activity in the amygdala of healthy subjects viewing fearful faces (Gamer et al, 2010; Kirsch et al, 2005; Petrovic et al, 2008), whereas the results were mixed for happy faces (Domes et al, 2007a; Gamer et al, 2010).

Patients with schizophrenia have been reported to have low oxytocin levels, which are associated with an increase in psychotic symptoms and fewer prosocial behaviors (Keri et al, 2009; Rubin et al, 2010). Oxytocin restored socially aberrant behaviors in an animal model of schizophrenia (Caldwell et al, 2008), suggesting a link between oxytocin and 
symptoms or social behaviors in schizophrenia. Indeed, recent studies have shown that oxytocin treatment decreased psychotic symptoms (Feifel et al, 2010; Pedersen et al, 2011) and improved emotion recognition (Fischer-Shofty et al, 2013; Pedersen et al, 2011), verbal memory (Feifel et al, 2012), and olfactory identification (MR Lee et al, 2013) in patients with schizophrenia.

Despite accumulated evidence on the effects of oxytocin on behavioral outcomes in schizophrenia, the effect of oxytocin on the neural activity underlying emotion processing in this disorder has not been investigated. The present study thus examined the effect of a single dose of intranasal oxytocin on amygdala activity in patients with schizophrenia using an implicit facial emotion perception paradigm. On the basis of previous findings implicating aberrant amygdala responses to neutral faces in patients with schizophrenia, a baseline condition was included as a control condition in our study. It was hypothesized that inhaled oxytocin would modulate amygdala activity evoked by emotional faces in patients with schizophrenia and control subjects. In addition, we assessed empathy and attachment style in the participants to determine whether oxytocin-related behaviors affected the oxytocin-induced neural effect. We predicted that the effect of oxytocin on the amygdala activity underlying facial emotion recognition would be modulated by oxytocinrelated behaviors.

\section{MATERIALS AND METHODS}

\section{Participants}

The study included 16 male patients with schizophrenia and 16 age-matched healthy male controls. The patients (mean age \pm SD: $32.0 \pm 7.8$ years) were recruited from the Seoul National University Hospital (SNUH) outpatient clinic and fulfilled the DSM-IV criteria for schizophrenia evaluated using the Structured Clinical Interview for DSM-IV (SCID; First et al, 1996). Patients were included if their symptoms had persisted for $\geqslant 1$ year and their dose and type of psychotropic drugs had been stable for at least 4 weeks. All patients were taking psychotropic drugs at the time of experiments (antipsychotics, $n=14$; benzodiazepines, $n=13$; selective serotonin reuptake inhibitors, $n=12$; beta-blocker, $n=3$; psychostimulant, $n=3$; anticonvulsant, $n=2$; antiepileptics, $n=1$ ). None of the patients had a comorbid Axis I disorder except one patient who had coexistent obsessivecompulsive disorder. The Positive and Negative Syndrome Scale (PANSS) was used to evaluate symptom severity (mean \pm SD: $12.1 \pm 3.1$ for positive symptoms; $16.5 \pm 5.8$ for negative symptoms; $30.6 \pm 8.2$ for general symptoms). (Supplementary Table 1 in the Supplement)

The age-matched healthy controls (mean age \pm SD: $31.3 \pm 7.6$ years) were recruited through internet advertisements and were screened for Axis I disorders using the non-patient edition of the structured clinical interview for DSM-IV (SCID-NP).

All participants were right handed with the exception of one patient who was left handed and one control participant who was ambidextrous. The exclusion criteria were mental retardation, brain injury, neurological illness, alcohol or substance abuse, and acetic acid allergy. Our study was approved by the Institutional Review Board of SNUH, and written informed consent was obtained from each participant and from the parent when the participant was under 20 years of age, after complete description of the study to the participant.

\section{Drug Preparation}

The oxytocin was prepared according to the protocol described in a previous study (Marsh et al, 2010) and prepared by the Department of Pharmacy in Seoul National University Hospital. The dose of intranasal oxytocin (40 IU) was determined according to results of our pilot study that was designed to investigate an efficient dose of oxytocin nasal spray for face emotion recognition in healthy Korean males.

\section{Procedure}

The study was a randomized, double-blind, placebo-controlled crossover design. Subjects underwent functional magnetic resonance imaging (fMRI) on two occasions 1 week apart. The order of the drug administration was counterbalanced. Subjects were instructed to abstain from alcohol, nicotine, caffeine, and physical exercise for $24 \mathrm{~h}$ before the experiment and to avoid food and drinks except water for $2 \mathrm{~h}$ before the experiment. Upon arrival, the subjects were administered the Relationship Style Questionnaires (RSQ; Griffin and Bartholomew, 1994) and Interpersonal Reactivity Index (IRI; Davis, 1983; Kang et al, 2009) to measure attachment style and empathy, respectively.

Under the supervision of an experimenter, the subjects self-administered 10 puffs of oxytocin (40 IU) or placebo contained in a nasal spray, switching nostrils at 30-60-s intervals $45 \mathrm{~min}$ before the fMRI scan. Participants completed the Positive and Negative Affect Schedule (PANAS; Watson et $a l, 1988$ ) and a visual analog scale (VAS) before administration of the drugs and after the fMRI session to examine mood changes throughout the experiment that possibly confound the effects of oxytocin (Figure 1).

The fMRI design was similar to that reported previously (Hall et al, 2008). With a block design, each block of six faces showing one emotion among fearful, happy and neutral expressions (three Korean females and three Korean males) were interspersed with baseline blocks (12 s) consisting of fixation cross hairs. The faces were selected from databases of ChaeLee Korean Facial Expression of Emotion (C-KFEE; $\mathrm{K}-\mathrm{U}$ Lee et al, 2013) and Korean Facial Expressions of Emotion (KOFEE) and the mean of accuracy rates for 40 University Korean students were 99\% in happy, 97\% in neutral, and $79 \%$ in fearful faces (see Supplementary Figure 1 in the Supplement for face stimuli). The face blocks began with the word 'Gender?' written in Korean for $1 \mathrm{~s}$. Each face stimulus was randomly presented in a block for $3 \mathrm{~s}$, followed by the fixation cross hairs $(0.5 \mathrm{~s})$. Each of the faces were presented once within a block and repeated across block. Participants were instructed to identify the gender of each face by pressing a button. The experiment consisted of two runs, in which each run consisted of three fearful $(22 \mathrm{~s}$ each; 6 trials per block), three happy (22 s each; 6 trials per block), and three neutral blocks ( $22 \mathrm{~s}$ each; 6 trials per block), and 10 baseline blocks (12 s each; see Supplementary Figure 1 in the Supplement for the fMRI task paradigm). The face blocks were presented in different pseudorandom orders across the 


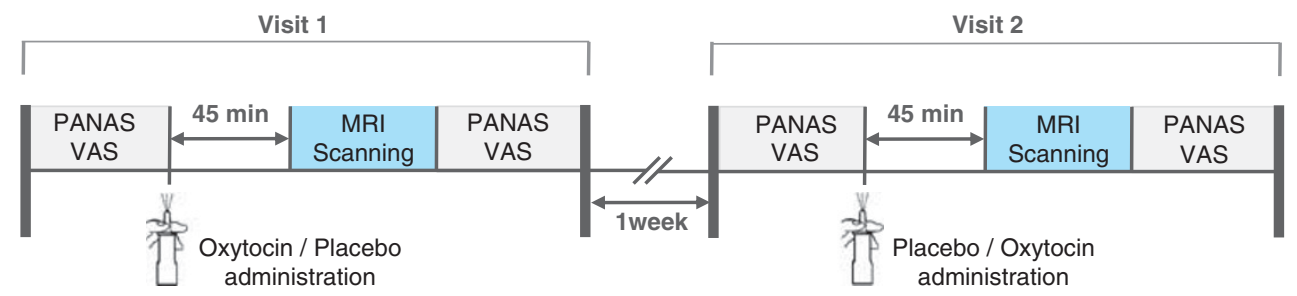

Figure I Experimental procedure. Subjects underwent the experiment on two occasions with either oxytocin or placebo I week apart. Functional magnetic resonance imaging ( $\mathrm{MMRI}$ ) session was commenced $45 \mathrm{~min}$ after drug application and the mood scales were performed before drug administration and after the MRI scanning. PANAS, Positive and Negative Affect Schedule; VAS, visual analog scale.

runs with a restriction that the same conditions do not occur in successive blocks. The same task was used in oxytocin and placebo conditions.

\section{Image Acquisition}

The blood oxygenation level-dependent (BOLD) signal was acquired using a 3T Siemens Trio MRI scanner (Siemens Healthcare, Erlangen, Germany) using T2-weighted gradient echo planar imaging (repetition time $[\mathrm{TR}]=2000 \mathrm{~ms}$; echo time $[\mathrm{TE}]=30 \mathrm{~ms}$; field of view $[\mathrm{FOV}]=220 \mathrm{~mm}$; flip angle $=90^{\circ} ; 4-\mathrm{mm}$ thickness; 27 axial slices; matrix $=64 \times$ 64). T1 anatomical volume reference images were also acquired $(\mathrm{TR}=1670 \mathrm{~ms} ; \mathrm{TE}=1.89 \mathrm{~ms} ; \mathrm{FOV}=250 \mathrm{~mm}$; flip angle $=9^{\circ}$; $1-\mathrm{mm}$ thickness; 208 slices; matrix $=256 \times 256$ ). Foam pads were used to reduce head motion. Data from one control subject were excluded from all analysis due to head motion $>3 \mathrm{~mm}$ in the MRI scanner. Data from one patient were also excluded from the analysis for the second run due to head motion.

\section{Image Preprocessing and Analysis}

Images were preprocessed and analyzed using Statistical Parametric Mapping 8 (SPM 8) software (http://www.fil.ion. ucl.ac.uk/spm/software/spm8/). Previous studies demonstrated maximal amygdala activation during the first run and amygdala habituation over time (Breiter et al, 1996; Hall et al, 2008). Therefore, our analysis was performed for each run to investigate oxytocin-elicited change during maximal BOLD signal and to examine change in the oxytocin-induced effect on amygdala activation over time.

The first three images were discarded to avoid unstable magnetic artifacts. The remaining images were spatially realigned to the first image for head-motion correction. The mean realigned functional image was normalized to the EPI template in the Montreal Neurological Institute (MNI) space and was resampled into $3-\mathrm{mm}^{3}$ voxels. Data were smoothed with an 8-mm full width at half-maximum (FWHM) Gaussian kernel. Contrast images were obtained for each emotion against baseline in each subject and entered in the second-level random-effects model for within- and between-group analysis. To investigate regions of significant difference between the groups at baseline, a two-sample $t$-test was performed for placebo condition. Clusters of activation were considered significant using height and extent thresholds of $p<0.05$ at the cluster level for multiple comparisons (Poline et al, 1997).

A region of interest (ROI) analysis was performed on the left and right amygdala regions in order to test our hypothesis of the effects of oxytocin on amygdala function. The ROIs for the bilateral amygdala were anatomically defined using WFU PickAtlas Version 2.4 (Maldjian et al, 2003). The mean percent BOLD signal change (PSC) was extracted from the ROIs for each face condition (fearful, happy, and neutral) and for the baseline using the MarsBaR toolbox (http://marsbar.sourceforge.net/) (Orme-Johnson et al, 2006). The mean PSC for the fearful, happy, or neutral face contrasts versus baseline were calculated. Effects of oxytocin on amygdala activation was tested using repeated measures analysis of variance (ANOVA) with drug (oxytocin, placebo), emotional valence (fearful, happy, neutral) and hemisphere (left, right) as within-subject factors and group (patients, controls) as a between-subjects factor. Post hoc $t$-tests were performed on significant interactions $(p<0.05$, two-tailed). The analyses with the mean PSC were performed using the IBM Statistical Package for the Social Sciences version 18 (SPSS).

Finally, to examine whether person's clinical variables and/or psychological scales are associated with amygdala functions in emotion processing, Pearson correlation analyses were conducted for each participant group. Further, a mixed ANOVA was used to analyze the mood scale scores with the PANAS or VAS subscores, drug (oxytocin, placebo) as withinsubject factors and with the group as between-subject factor to examine change in mood after drug administration. Independent $t$-test with Bonferroni correction for multiple comparisons was conducted to examine the group differences in attachment style and empathy.

\section{RESULTS}

\section{Behavioral Outcomes}

We found no significant main or interaction effect in the gender identification in the first, second, or full session. For mood change scores measured by subtracting pretreatment scores from posttreatment scores in the VAS and PANAS, a main effect of emotional valence was significant in the PANAS $\left(F_{1,29}=5.90, p=0.022\right)$, indicating reduction of negative affects after drug administration (see Supplementary Table 2 in the Supplement). Other effects were not found to be significant in the mood scales. The analysis of attachment style data revealed that secure attachment scores were significantly lower $\left(t_{29}=3.00, p=0.006\right)$ in patients with schizophrenia than control subjects after Bonferroni correction $(p=0.01)$. Regarding empathy, patients had significantly higher scores for personal distress $\left(t_{29}=-3.95\right.$, 
Table I Clusters of Significant Difference Between the Groups at Placebo Condition

\begin{tabular}{|c|c|c|c|c|c|c|c|}
\hline & & \multicolumn{3}{|c|}{ Coordinates } & \multirow[t]{2}{*}{ Cluster $\mathbf{k}$} & \multirow[t]{2}{*}{ Corrected $P$-value } & \multirow[t]{2}{*}{$t$-test } \\
\hline & & $x$ & y & $\mathbf{z}$ & & & \\
\hline Cerebellum & $L$ & -9 & -55 & -23 & 13719 & $<0.001$ & 5.10 \\
\hline \multicolumn{8}{|c|}{ Neutral face > baseline: patients $>$ healthy controls } \\
\hline \multicolumn{8}{|c|}{ Happy face > baseline: patients $>$ healthy controls } \\
\hline Cerebellum & $\mathrm{L}$ & -9 & -52 & -17 & 9455 & $<0.001$ & 4.99 \\
\hline
\end{tabular}

Abbreviations: $L$, left; $R$, right.

${ }^{\text {a S}}$ mall volume correction within amygdala region.

$p<0.001$ ) than did control subjects (see Supplementary Table 1 in the Supplement).

\section{Group Differences in Placebo Condition}

During the first run, patients with schizophrenia showed greater activation for fearful faces in left cerebellum than healthy controls in the placebo condition. However, amygdala activation did not significantly differ between the two groups in the same contrast (Table 1). Unlike the fearful $>$ baseline contrast, we found significant difference in amygdala activation between patient and control groups in the neutral faces $>$ baseline contrast. That is, patients with schizophrenia displayed an increased activation in the left amygdala $(p<0.05$ corrected within an amygdala ROI; Table 1$)$ and greater activation in the right superior orbitofrontal cortex than controls for the neutral faces in the placebo condition. For happy faces, the patient group had greater activation than in the control group in right cerebellum (Table 1).

\section{ROI Analyses}

Descriptive data of PSC for each emotion in each group are presented in Figure 2 and Supplementary Table 3 in the Supplement. When we performed analyses in the first run, a 2 (drug) $\times 3$ (emotional valence) $\times 2$ (hemisphere) $\times 2$ (group) ANOVA yielded a significant main effect of emotional valence $\left(F_{2,58}=4.98, p=0.010, \eta_{\mathrm{p}}^{2}=0.25\right)$, denoting less amygdala activity to neutral relative to emotional faces. No main effect of drug, group, or hemisphere in amygdala activities was found.

A significant interaction of the drug $\times$ group $\left(F_{1,29}=7.61\right.$, $\left.p=0.010, \eta_{\mathrm{p}}^{2}=0.21\right)$ was found in the first run, indicating differential effects of oxytocin between the patient and control groups (Figure 3b). Post hoc analysis was based on the contrasts of oxytocin $>$ placebo to compute oxytocininduced signal change and averaged PSCs across emotional valences were extracted from bilateral amygdala. Independent $t$-test revealed significant group differences in oxytocininduced signal change $\left(t_{29}=2.76, p=0.01\right)$, indicating that the patient group showed slightly attenuated activation following oxytocin treatment (mean $\pm \mathrm{SD} ;-0.03 \pm 0.10$;
Figure 3c), whereas the control group exhibited increased activation (mean $\pm \mathrm{SD} ; 0.71 \pm 0.10$; Figure $3 \mathrm{~d}$ ). We also found a significant drug $\times$ emotional valence interaction $\left(F_{2,58}=6.64, p=0.003, \eta_{\mathrm{p}}^{2}=0.32\right.$; Figure $\left.3 \mathrm{a}\right)$. For the post hoc analysis, the contrasts of oxytocin $>$ placebo were computed in a total sample and were compared for different emotional valences (between fearful and happy, fearful and neutral, or happy and neutral faces), using paired $t$-test. When applying a Bonferroni correction $(p=0.017)$, the comparison between fearful and happy faces was significant $\left(t_{30}=-3.72, p=0.001\right)$, indicating attenuated activation in response to the fearful faces and increased activation in response to happy faces (mean $\pm S D ;-0.06 \pm 0.17$ vs $0.11 \pm 0.21)$. An interaction of hemisphere $\times$ group was also significant $\left(F_{1,29}=4.58, p=0.041, \eta_{\mathrm{p}}^{2}=0.12\right)$. The post hoc independent $t$-test showed significantly more left amygdala activation in control than in patient groups $\left(t_{29}=2.10\right.$ $p=0.049$ ), but no significant difference in right-amygdala activation between the groups $\left(t_{29}=0.42 p=0.676\right)$. The findings were similar when one left-handed patient was excluded.

Additional analysis for the second run showed a significant interaction of drug $\times$ group $\left(F_{1,28}=5.21, p=0.030, \eta_{\mathrm{p}}^{2}=0.16\right)$. Any other main or interaction effects were not significant. When the analysis was performed for the full session, a significant effect was found only in the drug $\times$ group interaction $\left(F_{1,28}=7.46, p=0.011, \eta_{\mathrm{p}}^{2}=0.21\right)$.

\section{Correlations Between Amygdala Activity and Behavioral Outcomes Following Oxytocin Treatment}

The Pearson correlation analysis revealed a significant negative correlation between the oxytocin (happy-baseline) $>$ placebo $_{\text {(happy-baseline) }}$ contrast in the right amygdala during the first run and the RSQ preoccupied attachment score in control subjects $(r=-0.733, p=0.002$; Figure 4) after Bonferroni correction $(p=0.005)$, indicating less degree of activation increase following oxytocin in persons who have preoccupied attachment style. We did not find a significant correlation between oxytocin-related effects and symptom severity and antipsychotic doses in the patients. 

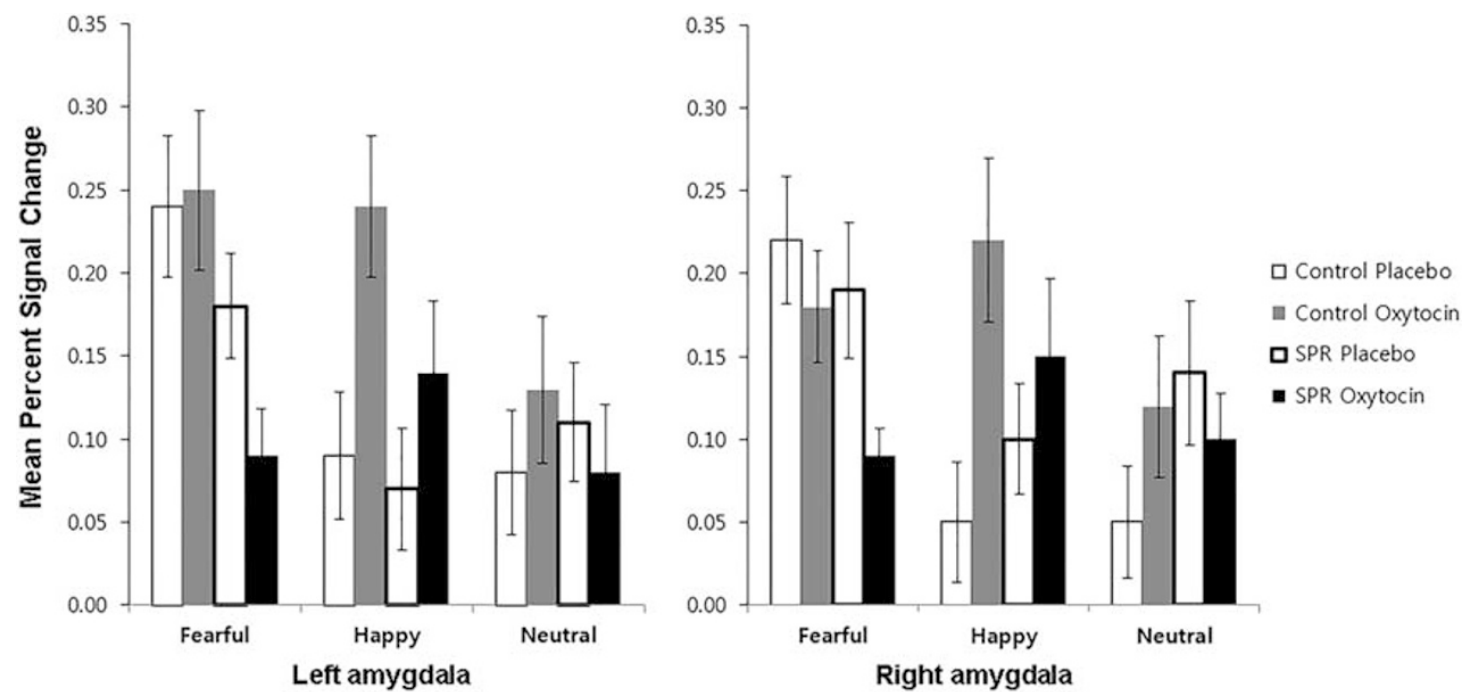

Figure 2 Percent blood oxygenation level-dependent (BOLD) signal changes for amygdala region of interests (ROls) for each emotion within each group in the first run. Data were extracted from anatomically defined amygdala regions for the contrast of fearful, happy, or neutral face versus baseline. Error bars indicate standard errors. SPR, schizophrenia.

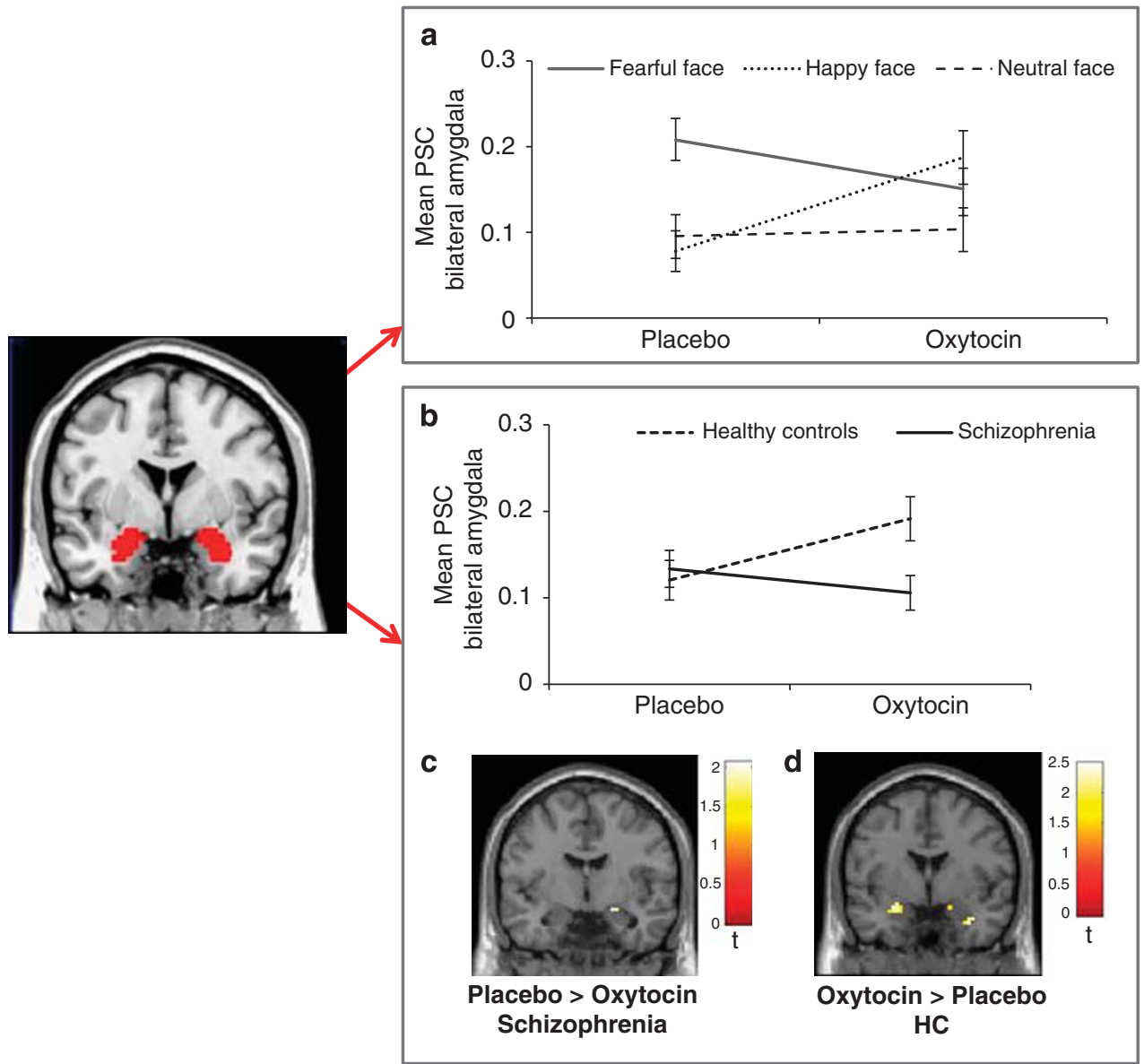

Figure 3 The effects of oxytocin on bilateral amygdala activity. (a) Differential effects of oxytocin on amygdala activity for different types of emotion in a total sample $(n=31)$ : oxytocin increased amygdala activation for happy faces and decreased activation for fearful faces. (b) Different oxytocin effects on amygdala activity between the patient and control groups in response to emotional faces. (c) Oxytocin-induced decrease of amygdala activation in response to emotional faces in schizophrenia. (d) Oxytocin-induced increase of activation in response to emotional faces in healthy controls. Threshold for t-maps was $p<0.05$ (uncorrected) for illustration purposes. Error bars indicate SEM. PSC, percentage of signal change. 


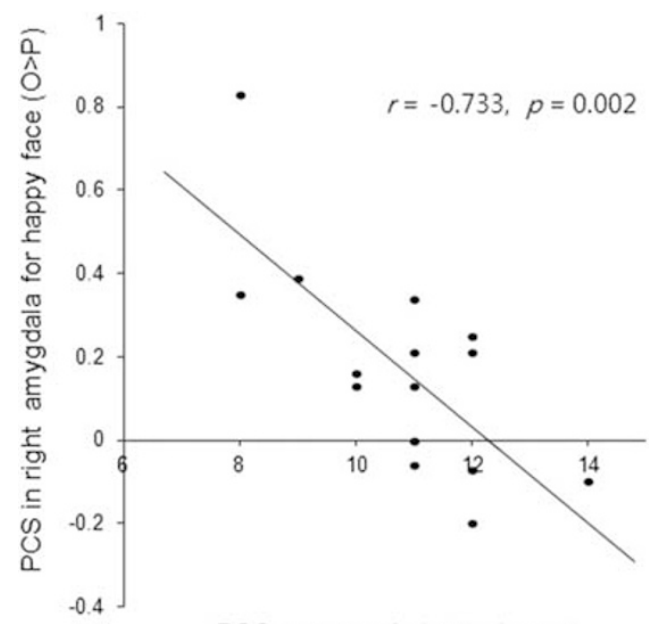

RSQ preoccupied attachment

Healthy Controls

Figure 4 Correlations of oxytocin-induced effects in right amygdala with attachment style in healthy controls. O>P, the contrast for oxytocinplacebo; PSC, percentage of signal change; RSQ, Relationship Style Questionnaire. The higher RSQ score indicates more preoccupied attachment style, which was associated with less oxytocin-induced increased activation in right amygdala in response to happy faces.

\section{DISCUSSION}

To our knowledge, the present study is the first to investigate the effect of intranasal oxytocin on brain activity related to facial emotion recognition in patients with schizophrenia using a within-subject design. In our data, intranasal oxytocin exerted differential effects on amygdala activity across emotional valences. Oxytocin decreased amygdala reactivity in response to fearful faces, whereas nasal spray increased amygdala activity for happy faces. In addition, oxytocin sniffs induced differential effects between the patient and control groups. Oxytocin attenuated amygdala activity in patients with schizophrenia, whereas neuropeptide augmented amygdala activity in healthy controls. These results suggest that oxytocin has a differential modulatory effect on amygdala response to emotional faces between patients with schizophrenia and Korean healthy individuals.

The inability to recognize facial emotion is a core deficit in schizophrenia (Morris et al, 2009). Meta-analytic studies demonstrated that dysfunction in several brain regions including amygdala, frontal and temporal regions underlies the deficit in emotion perception in this disorder (Taylor et al, 2012). With regard to amygdala activation, the findings are different according to which condition was used for contrasts. Reduced amygdala activity has been found when contrasts were made between valenced, especially fearful, and neutral faces in patient with schizophrenia. However, in a few studies that used baseline as a control condition, increased activity was found for both neural and fearful faces (Hall et al, 2008; Surguladze et al, 2006) or only for neutral faces in limbic regions (Holt et al, 2006). These results suggest overactive response to the neutral faces and/or general greater activity for emotional faces in patients with schizophrenia. In the current study, a significant difference in amygdala activation between the patients and control groups was found for neutral, but not for fearful and happy faces. These results correspond with the findings from the previous study that employed similar fMRI design with our study (Hall et al, 2008). Augmented amygdala response for neutral faces may reflect the tendency to perceive nonthreatening or nonsalient facial expressions as threatening or salient in patients with schizophrenia.

The present study demonstrated oxytocin-induced effect on amygdala reactivity for emotional expressions in patients with schizophrenia. This result is consistent with findings reported from a recent behavioral study showing oxytocin-related effect on recognition of emotional faces in patients with schizophrenia (Fischer-Shofty et al, 2013). Recently, research has shown that intranasal oxytocin induces functional changes in brain activity for social stimuli in specific psychiatric disorders including autism spectrum disorders (Guastella et al, 2010) and social anxiety disorder (Labuschagne et al, 2010, 2011). No studies have investigated the neural response to oxytocin in patients with schizophrenia. In our study, intranasal oxytocin attenuated amygdala activity for emotional faces in patients with schizophrenia. Previous neuroimaging studies have shown that intranasal oxytocin reduced amygdala reactivity for emotional faces in healthy males (Gamer et al, 2010; Kirsch et al, 2005; Petrovic et al, 2008) and patients with social anxiety disorder (Labuschagne et al, 2010), although the results were mixed (Domes et al, 2007a; Gamer et al, 2010). It has been proposed that reduction of social anxiety and silence is likely to underlie effects of oxytocin on amygdala attenuation for emotional processing (Bartz et al, 2011). Increased sensitivity to threatening or neutral social stimuli has been suggested in patients with schizophrenia (Green et al, 2003; Surguladze et al, 2006). In line with this, one of plausible explanations for our results could be that inhaled oxytocin may reduce social anxiety and sensitivity to emotional faces in patients with schizophrenia. Given a limited effect of current antipsychotic medications on the impaired ability to perceive facial emotion in patients with schizophrenia (Penn et al, 2009), oxytocin nasal spray may be effective in altering dysfunctional neural activity related to emotional processing in those with schizophrenia.

Previous studies of emotion-related changes by oxytocin in amygdala activity have reported conflicting results in healthy individuals. We found that oxytocin administration increased amygdala activity in response to emotional faces in healthy controls. Several studies have reported an oxytocin-induced reduction in amygdala activity in response to fearful emotion in male subjects (Bethlehem et al, 2013), and a single study observed an oxytocin-induced increase in amygdala activity in response to fearful emotion in female participants (Domes et al, 2009). Furthermore, one previous study observed an increase (Gamer et al, 2010) and another found a decrease (Domes et al, 2007a) in amygdala activity in response to happy emotion. These disparate findings may be attributable to an influence of context or to individual differences on the relationship between oxytocin and social cognition, as proposed by Bartz et al (2011). The effect of oxytocin has been reported to be restricted to individuals with specific traits (Bartz et al, 2010b; Singer et al, 2008) and moderated by task-related properties such as difficulty (Bartz et al, 2010b; Domes et al, 2007b). Indeed, the greater oxytocin-induced increase in amygdala activity in response to happy faces in the healthy controls in our study was strongly associated with the lower preoccupied attachment 
style score. This result implies that oxytocin-induced effect on amygdala activity is likely to be weakened in persons with attachment pattern of high anxiety and low avoidance (Griffin and Bartholomew, 1994). Our finding agrees with previous observations involving the influence of parentallove withdrawal (Huffmeijer et al, 2012) and anxious attachment (Bartz et al, 2010a) on the oxytocin effect. Further, the influence of cultural factors on our results cannot be ruled out. Distinct differences in the recognition of facial expressions (Matsumoto, 1992) and the role of oxytocin receptor genes in emotional processing (Kim et al, 2011) have been reported in Asian and Western subjects. Future studies are needed to clarify the impact of cultural differences on the oxytocin nasal spray-induced effect on social cognition.

In our data, the effects of oxytocin on amygdala for facial expression were different between the schizophrenia and control groups. Oxytocin increased amygdala activation in the patient group, whereas the peptide decreased activation in the control group. Given the amygdala activation levels were similar in both groups at baseline (placebo condition), it does not seem that the oxytocin-induced changes may imply a recovery of amygdala function in the patients. Instead, they are likely to reflect distinct roles of intranasal oxytocin in the patients with schizophrenia and Korean healthy subjects. Prior studies have suggested that the effects of oxytocin on social cognition may depend on individual differences (Vrtička et al, 2008) and social context (De Dreu et al, 2010). In particular, affiliative motivation, personal significance, and perceptual selectivity to social stimuli have been indicated as important moderators of diverse oxytocininduced effects (Bartz et al, 2011; Vrtička et al, 2008). In this respect, it can be assumed that different psychological and biological characteristics between the patient and control groups, such as sensitivity to specific emotions, might affect the response to oxytocin. Although our data failed to show significant three-way interaction between drug, group and emotional valence, a close look at our data (Figure 2 and Supplementary Table 3 in the Supplement) makes it possible to speculate that the oxytocin-induced changes were strongly driven by specific emotion, for example a decreased amygdala activity in the patients by fearful and neutral faces and an increased activity in the control subjects by happy faces following oxytocin treatment. In this regard, we cautiously suggest that this might reflect the group-specific effect of oxytocin on personally meaningful and motivated emotions. More studies are needed to explore psychological and behavioral mechanism underpinning the differential effects of oxytocin between the schizophrenic and healthy individuals to draw a firm conclusion.

Consistent with previous observation (Adolphs, 2002; Breiter et al, 1996; Hall et al, 2008), amygdala response to emotional faces habituated over time. The differential oxytocin-related effects across emotional valences shown in the first run were weakened in the second run. However, the differential effects of hormone between the groups shown in the first run were apparent in the second run as well. This suggests that differential effects of oxytocin on amygdala activity between patients and control subjects are robust enough to resist habituation despite general amygdala habituation over time in emotion recognition.

Our study has some limitations. First, all patients participated in this study were taking psychotropic drugs at the time of the experiment. Although no statistical significant correlation between antipsychotic drugs and oxytocin effect on amygdala activity was observed in our data, a potential interaction between the psychotropic drugs and intranasal oxytocin may influence the results. Prior studies have suggested that biological function of oxytocin is moderated by dopaminergic system in animals (Bale et al, 2001; Liu and Wang, 2003). Second, accuracy rate for the fearful face stimuli was lower than that for happy and neutral face stimuli, which may not have evoked sufficiently strong neural responses to fearful faces. Third, we did not assess socioeconomic status (SES) of subjects and their parents. Especially, parental SES can be an important factor that influences cognitive functioning in patients with schizophrenia (Yeo et al, 2014). Lastly, we enrolled only male participants to exclude an interaction between exogenous and endogenous oxytocin. Further studies are needed to assess the effect of oxytocin on emotion recognition in female patients with schizophrenia.

In conclusion, our study showed that oxytocin reduced amygdala activity in response to emotional faces in patients with schizophrenia, whereas oxytocin increased amygdala activity in healthy control subjects. An influence of attachment style on oxytocin-related neural change in control subjects implicates that individual difference or cultural factors should be carefully considered in future research. Our data, together with previous findings showing a beneficial effect of oxytocin for psychiatric disorders such as autism (Domes et al, 2013; Watanabe et al, 2014) and social anxiety (Labuschagne et al, 2010; Labuschagne et al, 2011), provide new evidence supporting a modulatory effect of oxytocin on neural response to emotion processing for patients with schizophrenia. It seems that a single dose of oxytocin causes a subtle change in brain activity of stabilized schizophrenia patients who have taken psychotropic medications. However, even a small change in brain activity can alter the behavioral outcomes. Future studies are needed to investigate the effect of daily administration of intranasal oxytocin on neural activity and to compare the effectiveness of different doses in patients with schizophrenia.

\section{FUNDING AND DISCLOSURE}

JSK's work has been funded by Basic Science Research Program through the National Research Foundation of Korea (NRF) funded by the Ministry of Science, ICT and Future Planning (2013R1A2A1A03071089). The remaining authors declare no conflict of interest.

\section{ACKNOWLEDGMENTS}

We thank Ye Seul Shin MS and Da-Jung Shin MS for their research assistance and support (SNU-MRC, Seoul, Republic of Korea) as well as all the participants of this study.

\section{REFERENCES}

Adolphs R (2002). Neural systems for recognizing emotion. Curr Opin Neurobiol 12: 169-177.

Aleman A, Kahn RS (2005). Strange feelings: do amygdala abnormalities dysregulate the emotional brain in schizophrenia? Prog Neurobiol 77: 283-298. 
Bale TL, Davis AM, Auger AP, Dorsa DM, McCarthy MM (2001). CNS region-specific oxytocin receptor expression: importance in regulation of anxiety and sex behavior. J Neurosci 21: 2546-2552.

Bartz JA, Simeon D, Hamilton H, Kim S, Crystal S, Braun A et al (2010a). Oxytocin can hinder trust and cooperation in borderline personality disorder. Soc Cogn Affect Neurosci 6: 556-563.

Bartz JA, Zaki J, Bolger N, Hollander E, Ludwig NN, Kolevzon A et al (2010b). Oxytocin selectively improves empathic accuracy. Psychol Sci 21: 1426-1428.

Bartz JA, Zaki J, Bolger N, Ochsner KN (2011). Social effects of oxytocin in humans: context and person matter. Trends Cogn Sci 15: 301-309.

Bethlehem RAI, van Honk J, Auyeung B, Baron-Cohen S (2013). Oxytocin, brain physiology, and functional connectivity: a review of intranasal oxytocin fMRI studies. Psychoneuroendocrinology 38: 962-974.

Breiter HC, Etcoff NL, Whalen PJ, Kennedy WA, Rauch SL, Buckner RL et al (1996). Response and habituation of the human amygdala during visual processing of facial expression. Neuron 17: 875-887.

Caldwell HK, Stephens SL, Young WS (2008). Oxytocin as a natural antipsychotic: a study using oxytocin knockout mice. Mol Psychiatry 14: 190-196.

Davis MH (1983). Measuring individual differences in empathy: evidence for a multidimensional approach. J Pers Soc Psychol 44: 113.

De Dreu CK, Greer LL, Handgraaf MJJ, Shalvi S, Van Kleef GA, Baas $M$ et al (2010). The neuropeptide oxytocin regulates parochial altruism in intergroup conflict among humans. Science 328: $1408-1411$.

Domes G, Heinrichs M, Gläscher J, Büchel C, Braus DF, Herpertz SC (2007a). Oxytocin attenuates amygdala responses to emotional faces regardless of valence. Biol Psychiatry 62: 1187-1190.

Domes G, Heinrichs M, Kumbier E, Grossmann A, Hauenstein K, Herpertz SC (2013). Effects of intranasal oxytocin on the neural basis of face processing in autism spectrum disorder. Biol Psychiatry 74: 164-171.

Domes G, Heinrichs M, Michel A, Berger C, Herpertz S (2007b). Oxytocin improves mindreading in humans. Biol Psychiatry 61: 731-733.

Domes G, Lischke A, Berger C, Grossmann A, Hauenstein K, Heinrichs $\mathrm{M}$ et al (2009). Effects of intranasal oxytocin on emotional face processing in women. Psychoneuroendocrinology 35: 83-93.

Feifel D, MacDonald K, Cobb P, Minassian A (2012). Adjunctive intranasal oxytocin improves verbal memory in people with schizophrenia. Schizophr Res 139: 207-210.

Feifel D, Macdonald K, Nguyen A, Cobb P, Warlan H, Galangue B et al (2010). Adjunctive intranasal oxytocin reduces symptoms in schizophrenia patients. Biol Psychiatry 68: 678-680.

First MB, Spitzer RL, Gibbson M, Williams JBW (1996). Structured Clinical Interview for DSM-IV Axis I disorders. New York State Psychiatric Institute: New York.

Fischer-Shofty M, Shamay-Tsoory SG, Levkovitz Y (2013). Characterization of the effects of oxytocin on fear recognition in patients with schizophrenia and in healthy controls. Frontiers in Neuroscience 7: 127.

Fitzgerald D, Angstadt M, Jelsone L, Nathan P, Phan K (2006). Beyond threat: amygdala reactivity across multiple expressions of facial affect. Neuroimage 30: 1441-1448.

Gamer M, Zurowski B, Buchel C (2010). Different amygdala subregions mediate valence-related and attentional effects of oxytocin in humans. Proc Natl Acad Sci USA 107: 9400-9405.

Green M, Williams L, Davidson D (2003). Visual scanpaths to threat-related faces in deluded schizophrenia. Psychiatry Res 119: 271-285.

Griffin D, Bartholomew K (1994). Models of the self and other: fundamental dimensions underlying measures of adult attachment. J Pers Soc Psychol 67: 430-445.
Guastella AJ, Einfeld SL, Gray KM, Rinehart NJ, Tonge BJ, Lambert TJ et al (2010). Intranasal oxytocin improves emotion recognition for youth with autism spectrum disorders. Biol Psychiatry 67: 692-694.

Gur RE, McGrath C, Chan RM, Schroeder L, Turner T, Turetsky BI et al (2002). An fMRI study of facial emotion processing in patients with schizophrenia. Am J Psychiatry 159: 1992-1999.

Hall J, Whalley H, McKirdy J, Romaniuk L, McGonigle D, McIntosh A et al (2008). Overactivation of fear systems to neutral faces in schizophrenia. Biol Psychiatry 64: 70-73.

Holt D, Kunkel L, Weiss A, Goff D, Wright C, Shin L et al (2006). Increased medial temporal lobe activation during the passive viewing of emotional and neutral facial expressions in schizophrenia. Schizophr Res 82: 153-162.

Huffmeijer R, Alink LR, Tops M, Bakermans-Kranenburg MJ, van IJzendoorn $\mathrm{MH}$ (2012). Asymmetric frontal brain activity and parental rejection predict altruistic behavior: moderation of oxytocin effects. Cogn Affect Behav Neurosci 12: 382-392.

Kang I, Kee S-W, Kim S-E, Jeong B, Hwang J-H, Song J-E et al (2009). Reliability and validity of the Korean version of Interpersonal Reactivity Index. J Korean Neuropsychiatr Assoc 48: 352-358.

Keri S, Kiss I, Kelemen O (2009). Sharing secrets: oxytocin and trust in schizophrenia. Soc Neurosci 4: 287-293.

Kim HS, Sherman DK, Mojaverian T, Sasaki JY, Park J, Suh EM et al (2011). Gene-culture interaction oxytocin receptor polymorphism (OXTR) and emotion regulation. Soc Psychol Personal Sci 2: 665-672.

Kirsch P, Esslinger C, Chen Q, Mier D, Lis S, Siddhanti S et al (2005). Oxytocin modulates neural circuitry for social cognition and fear in humans. J Neurosci 25: 11489.

Labuschagne I, Phan KL, Wood A, Angstadt M, Chua P, Heinrichs M et al (2010). Oxytocin attenuates amygdala reactivity to fear in generalized social anxiety disorder. Neuropsychopharmacology 35: 2403-2413.

Labuschagne I, Phan KL, Wood A, Angstadt M, Chua P, Heinrichs M et al (2011). Medial frontal hyperactivity to sad faces in generalized social anxiety disorder and modulation by oxytocin. Int J Neuropsychopharmacol 15: 883-896.

Lee K-U, Kim J, Yeon B, Kim S-H, Chae J-H (2013). Development and standardization of extended ChaeLee Korean Facial Expressions of Emotions. Psychiatry Investigation 10: 155-163.

Lee MR, Wehring HJ, McMahon RP, Linthicum J, Cascella N, Liu F et al (2013). Effects of adjunctive intranasal oxytocin on olfactory identification and clinical symptoms in schizophrenia: results from a randomized double blind placebo controlled pilot study. Schizophr Res 145: 110-115.

Liu Y, Wang Z (2003). Nucleus accumbens oxytocin and dopamine interact to regulate pair bond formation in female prairie voles. Neuroscience 121: 537-544.

Maldjian JA, Laurienti PJ, Kraft RA, Burdette JH (2003). An automated method for neuroanatomic and cytoarchitectonic atlas-based interrogation of fMRI data sets. Neuroimage 19: 1233-1239.

Marsh AA, Yu HH, Pine DS, Blair RJ (2010). Oxytocin improves specific recognition of positive facial expressions. Psychopharmacology (Berl) 209: 225-232.

Matsumoto D (1992). American-Japanese cultural differences in the recognition of universal facial expressions. J Corss Cult Psychol 23: 72-84.

Michalopoulou PG, Surguladze S, Morley LA, Giampietro VP, Murray RM, Shergill SS (2008). Facial fear processing and psychotic symptoms in schizophrenia: functional magnetic resonance imaging study. Br J Psychiatry 192: 191-196.

Morris RW, Weickert CS, Loughland C (2009). Emotional face processing in schizophrenia. Curr Opin Psychiatry 22: 140-146.

Orme-Johnson DW, Schneider RH, Son YD, Nidich S, Cho ZH (2006). Neuroimaging of meditation's effect on brain reactivity to pain. Neuroreport 17: 1359-1363. 
Pedersen CA, Gibson CM, Rau SW, Salimi K, Smedley KL, Casey RL et al (2011). Intranasal oxytocin reduces psychotic symptoms and improves Theory of Mind and social perception in schizophrenia. Schizophr Res 132: 50-53.

Penn DL, Keefe RS, Davis SM, Meyer PS, Perkins DO, Losardo D et al (2009). The effects of antipsychotic medications on emotion perception in patients with chronic schizophrenia in the CATIE trial. Schizophr Res 115: 17-23.

Petrovic P, Kalisch R, Singer T, Dolan RJ (2008). Oxytocin attenuates affective evaluations of conditioned faces and amygdala activity. J Neurosci 28: 6607-6615.

Poline J-B, Worsley KJ, Evans AC, Friston KJ (1997). Combining spatial extent and peak intensity to test for activations in functional imaging. Neuroimage 5: 83-96.

Rubin LH, Carter CS, Drogos L, Pournajafi-Nazarloo H, Sweeney JA, Maki PM (2010). Peripheral oxytocin is associated with reduced symptom severity in schizophrenia. Schizophr Res 124: 13-21.

Shahrestani S, Kemp AH, Guastella AJ (2013). The impact of a single administration of intranasal oxytocin on the recognition of basic emotions in humans: a meta-analysis. Neuropsychopharmacology 38: 1929-1936.

Singer T, Snozzi R, Bird G, Petrovic P, Silani G, Heinrichs M et al (2008). Effects of oxytocin and prosocial behavior on brain responses to direct and vicariously experienced pain. Emotion 8: 781-791.
Surguladze S, Russell T, Kucharska-Pietura K, Travis M, Giampietro V, David A et al (2006). A reversal of the normal pattern of parahippocampal response to neutral and fearful faces is associated with reality distortion in schizophrenia. Biol Psychiatry 60: 423-431.

Taylor SF, Kang J, Brege IS, Tso IF, Hosanagar A, Johnson TD (2012). Meta-analysis of functional neuroimaging studies of emotion perception and experience in schizophrenia. Biol Psychiatry 71: 136-145.

Vrtička P, Andersson F, Grandjean D, Sander D, Vuilleumier P (2008). Individual attachment style modulates human amygdala and striatum activation during social appraisal. PLoS One 3: e2868.

Watanabe T, Abe O, Kuwabara $\mathrm{H}$, Yahata N, Takano Y, Iwashiro $\mathrm{N}$ et al (2014). Mitigation of sociocommunicational deficits of autism through oxytocin-induced recovery of medial prefrontal activity: a randomized trial. JAMA Psychiatry 71: 166-175.

Watson D, Clark LA, Tellegen A (1988). Development and validation of brief measures of positive and negative affect: the PANAS scales. J Pers Soc Psychol 54: 1063-1070.

Yeo RA, Martinez D, Pommy J, Ehrlich S, Schulz SC, Ho B-C et al (2014). The impact of parent socio-economic status on executive functioning and cortical morphology in individuals with schizophrenia and healthy controls. Psychol Med 44: 1257-1265.

Supplementary Information accompanies the paper on the Neuropsychopharmacology website (http://www.nature.com/npp) 